\title{
Disturbance Aspects of Iterative Learning Control
}

\author{
Mikael Norrlöf, Svante Gunnarsson \\ Division of Automatic Control \\ Department of Electrical Engineering \\ Linköpings universitet, SE-581 83 Linköping, Sweden \\ WWW: http://www. control.isy.liu.se \\ Email: mino@isy.liu.se, svante@isy.liu.se
}

17th August 2001

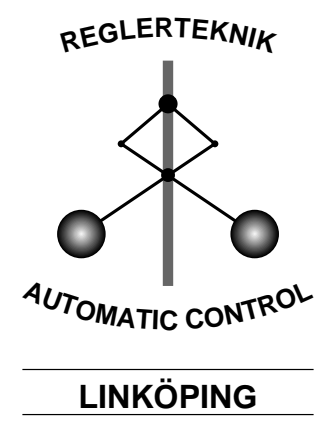

Report No.: LiTH-ISY-R-2371

Submitted to Engineering applications of Artificial Intelligence 14 (2001) 87-94

Technical reports from the Automatic Control group in Linköping are available by anonymous ftp at the address ftp.control.isy.liu.se. This report is contained in the file 2371 .pdf. 


\begin{abstract}
Disturbance aspects of Iterative Learning Control (ILC) are considered. By using a linear framework it is possible to investigate the influence of the disturbances in the frequency domain. The effects of the design filters in the ILC algorithm on the disturbance properties can then be analyzed. The analysis is supported by simulations and experiments.
\end{abstract}

Keywords: Robot control, iterative learning control, disturbance effects 


\title{
Disturbance Aspects of Iterative Learning Control ${ }^{\star}$
}

\author{
Mikael Norrlöf, Svante Gunnarsson ${ }^{1}$ \\ Department of Electrical Engineering, Linköping University, \\ SE-58183 Linköping, Sweden
}

\begin{abstract}
Disturbance aspects of Iterative Learning Control (ILC) are considered. By using a linear framework it is possible to investigate the influence of the disturbances in the frequency domain. The effects of the design filters in the ILC algorithm on the disturbance properties can then be analyzed. The analysis is supported by simulations and experiments.
\end{abstract}

Key words: Robot control; iterative learning control; disturbance effects

‡ This work was supported by CENIIT at Linköping University and ABB Robotics within ISIS at Linköping University.

1 Corresponding author. E-mail address: svante@isy.liu.se; Fax:+46-13-282622; Tel: $+46-13-281747$ 


\section{Introduction}

The word learning has many interpretations in engineering in general and in control in particular. In the control area learning in general means a procedure where a representation of a dynamic system or a strategy for controlling a dynamic system is adapted in some way in order to improve the overall performance.

In Iterative Learning Control (ILC) the assumption is that the control system is supposed to carry out the same operation repeatedly. This is a common situation in many robotics applications where a robot is supposed to do the same action, e.g. a welding or cutting operation, over and over again. Applications in other parts of manufacturing can also be found, but industrial robots have been the major application area for ILC. By using experience from one cycle the idea is to adjust the input signal in an appropriate way such that the performance of the system in the next cycle is improved. By measuring e.g the path error of a robot movement in one cycle the joint torques in the next cycle are adjusted such that the path error is reduced. An important difference between ILC and e.g. adaptive control or neural network modeling is that the whole input signal is adapted in ILC while the parameters in a parameterized representation of the controller or the model of the system is adapted in the other cases. ILC is a feed-forward (open loop) control method which means that the whole input sequence is precomputed before the cycle begins.

The standard assumption in ILC is that each cycle is carried out during a finite time interval $t=0, \ldots, N$. Since the implementation is done using a computer the problem is studied in discrete time. In order to cover a wide range of situations the following general linear system description will be used

$$
z_{k}(t)=T_{r}(q) r(t)+T_{u}(q) u_{k}(t)+T_{d}(q) d_{k}(t)+T_{n}(q) n_{k}(t)
$$

where $z_{k}(t), r(t)$ and $u_{k}(t)$ denote the system output signal, the reference signal and the ILC input signal respectively. The variables $d_{k}(t)$ and $n_{k}(t)$ denote load disturbance and measurement disturbance respectively. The aim in the paper is to analyze the effects of the disturbances on the overall system performance. A further step would be to design the ILC algorithm using a description of the statistical properties of the disturbance signals. The subscript $k$ denotes iteration (cycle) number. The reference signal $r(t)$ is the same in all iterations, which means that the whole sequence is known before the first iteration begins. All the other signals will change from iteration to iteration. The variables $T_{r}(q), T_{u}(q), T_{d}(q)$ and $T_{n}(q)$ denote stable discrete time filters. It is of course a restriction to confine the problem to linear systems, but on the other hand this will make it possible to obtain frequency domain insight into the disturbance properties of ILC. In many situations such insight is useful from an engineering viewpoint. 
The formulation in equation (1) is taken from (Norrlöf 1998), and it covers a wide class of situations ranging from an open loop control problem to a closed loop system operating under both feed-back and feed-forward control. In (Arimoto et al. 1984), which is often referred to as one of the original papers in the field of ILC, the ILC algorithm was used to generate the input to the system directly. In the framework here this corresponds to

$$
T_{u}=G \quad T_{r}=0 \quad T_{n}=0 \quad T_{d}=0
$$

where $G$ is the transfer function of the system to be controlled. In (2) and the sequel the argument of the involved transfer functions will sometimes omitted for convenience. The case that will be considered in this paper is depicted in Figure 1, where the signal $u_{k}(t)$ represents a signal added to the reference signal normally generated in the control system. Hence ILC is used as a complement to the conventional robot control system.

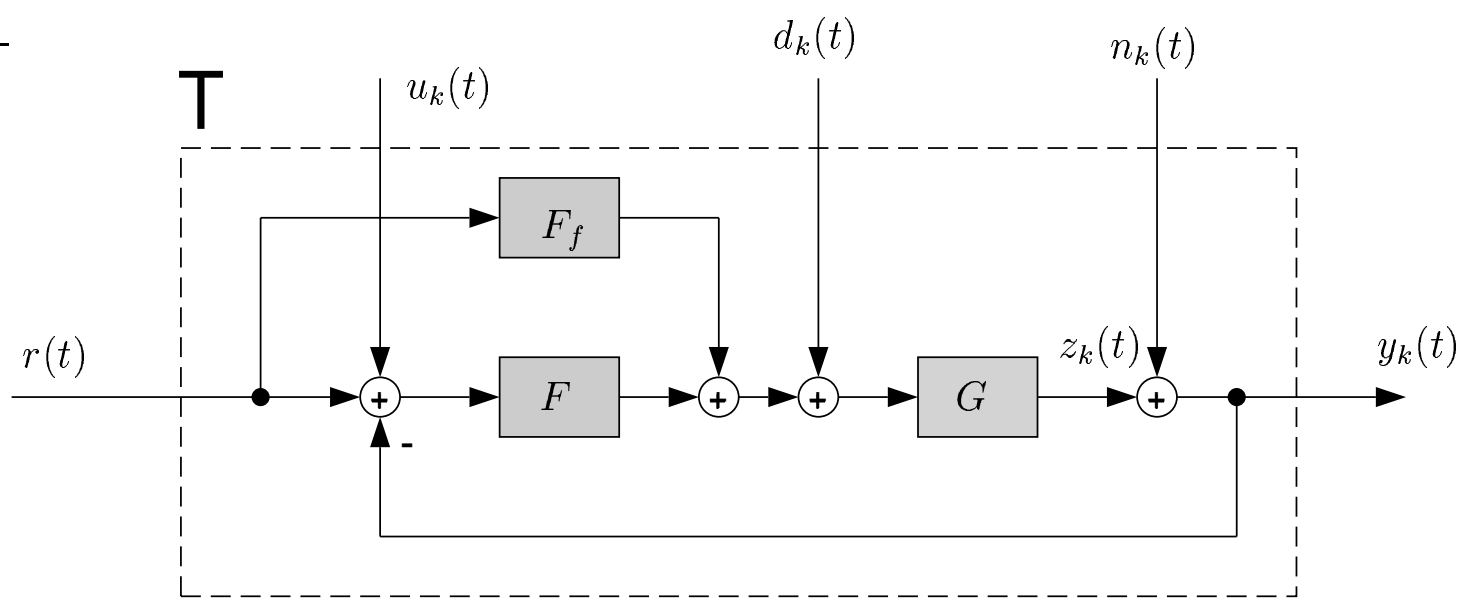

Fig. 1. An example of a realization of the system in equation (1).

The structure depicted in Figure 1 corresponds to the situation

$$
T_{r}=\frac{\left(F+F_{f}\right) G}{1+F G} \quad T_{u}=\frac{F G}{1+F G} \quad T_{d}=\frac{G}{1+F G} \quad T_{n}=\frac{-F G}{1+F G}
$$

where $G, F$ and $F_{f}$ are the transfer functions of the system to be controlled and the feed-back and feed-forward regulators respectively. A slight modification of the system structure shown in Figure 1 is to let the ILC input signal be used as a feed-forward signal added to the control signal generated by the feed-back and feed-forward parts of the controller. This just corresponds to a redefinition of the transfer function $T_{u}(q)$ in equation (1).

The fundamental problem in ILC is to design an update algorithm for the input signal $u_{k}(t)$ such that the error

$$
e_{k}(t)=r(t)-z_{k}(t)
$$


is reduced in some appropriate sense as the iterations proceed.

A general formula for updating the ILC input signal is given by

$$
u_{k+1}(t)=Q(q)\left(u_{k}(t)+L(q) \bar{e}_{k}(t)\right.
$$

where $\bar{e}_{k}(t)$ denotes the measured error signal i.e.

$$
\bar{e}_{k}(t)=r(t)-y_{k}(t)=e_{k}(t)-n_{k}(t)
$$

and $Q$ and $L$ are linear, possibly non-causal, filters. Since $u_{k+1}(t)$ is computed off-line, with both $\bar{e}_{k}(t)$ and $u_{k}(t)$ given over the whole time interval, noncausal filtering can be carried out.

ILC has been an active research are for almost two decades and numerous publications have been published. Among earlier contributions one finds (Craig 1988), (Hideg 1992), (Moore 1993) and (Horowitz 1993), while recent surveys of the area of ILC are given in (Moore 1998) and (Bien and Xu 1998). The results presented in the paper are extensions of the results presented in (Gunnarsson and Norrlöf 1997) and (Norrlöf 1998). The main contribution in this paper is the frequency domain analysis of the disturbance properties of ILC.

The paper is organized as follows. In Section 2 the ILC algorithm properties in general are discussed, while in Sections 3 and 4 the effects of load and measurement disturbances are investigated. Section 5 then contains simulations that supports the theoretical analysis. Experiments carried out on an industrial robot are then presented in Section 6. Finally some conclusions are given in Section 7.

\section{Algorithm Properties}

The question is now the following. Given the system defined by equation (1), how shall the filters $Q$ and $L$ in the update equation (5) be chosen such that the error $e_{k}(t)$ decreases in an appropriate way?

The main issues when choosing $Q$ and $L$ are convergence, robustness and influence of disturbances. In this paper the convergence and robustness issues will be mentioned briefly while the main attention is on disturbance aspects. While the earliest papers on ILC considered situations where almost no knowledge of the system to be controlled was available the use of some kind of a priori model in the design of ILC algorithms has become more and more common. An example of a model based method for choosing appropriate filters in the update equation is presented in (de Roover 1996) where methods for robust 
control design are applied and the filters are designed to give a convergent ILC algorithm despite uncertainties in the process model. In e.g. (Gorinevsky et al. 1995) the ILC input signal is formulated as an optimization problem, using an a priori model, resulting in a time domain updating equation for the input signal. In (Norrlöf 1998) it is shown how system identification can be used to build a low order linear model of an industrial robot which then is used for design of an appropriate ILC algorithm, which is also tested in experiments.

\subsection{Error equation}

In this section the aim is to derive the fundamental error equation that describes how the error evolves as the iterations proceed. The derivation of the error updating equation is formulated as the following lemma.

Lemma: Let $\tilde{e}$ be defined as

$$
\tilde{e}=\left(1-T_{r}\right) r
$$

i.e. the disturbance free error signal obtained without any ILC input, i.e. when $u_{0} \equiv 0$. Then the error signal is updated according to

$$
\begin{aligned}
e_{k+1} & =Q\left(1-L T_{u}\right) e_{k}+(1-Q) \tilde{e}+T_{d}\left(Q d_{k}-d_{k+1}\right) \\
& +T_{n}\left(Q n_{k}-n_{k+1}\right)+Q L T_{u} n_{k}
\end{aligned}
$$

Proof: Combining equations (4), (1), and (5) gives

$$
e_{k+1}=\tilde{e}-T_{u} Q u_{k}-T_{u} Q L e_{k}+T_{u} Q L n_{k}-T_{d} d_{k+1}-T_{n} n_{k+1}
$$

Using that

$$
-T_{u} u_{k}=e_{k}-\tilde{e}+T_{d} d_{k}+T_{n} n_{k}
$$

gives

$$
\begin{aligned}
e_{k+1} & =Q\left(1-L T_{u}\right) e_{k}+(1-Q) \tilde{e}+T_{d}\left(Q d_{k}-d_{k+1}\right) \\
& +T_{n}\left(Q n_{k}-n_{k+1}\right)+Q L T_{u} n_{k}
\end{aligned}
$$

which is the desired result.

The difference equation (8) has three types of driving terms. The first term is $\tilde{e}$ i.e. the error obtained when there is no ILC present. The other driving terms come from the load and measurement disturbances respectively, and they enter the equation in slightly different ways. The long run effects on 
the error of these terms will be analyzed in Sections 3 and 4 below. A result corresponding to equation (8) is presented in (Panzieri and Ulivi 1995) for the open loop case and for load disturbances only.

\subsection{Convergence condition}

The convergence properties are determined by the homogeneous part of the difference equation (8) and a sufficient condition for convergence is that

$$
\left|Q\left(e^{i \omega T}\right)\right|\left|1-L\left(e^{i \omega T}\right) T_{u}\left(e^{i \omega T}\right)\right|<1 \quad \forall \omega
$$

which means that

$$
\left|1-L\left(e^{i \omega T}\right) T_{u}\left(e^{i \omega T}\right)\right|<\frac{1}{\left|Q\left(e^{i \omega T}\right)\right|} \quad \forall \omega
$$

This criterion can be viewed in a Nyquist diagram, and with $Q=1$ the condition is that the Nyquist curve $L\left(e^{i \omega T}\right) T_{u}\left(e^{i \omega T}\right)$ should be held within a unit circle with center in +1 . On the other hand, by letting $Q$ be a filter with gain less than one, in some frequency range, the stability region can be increased. The prize paid for the increased stability region is that the error can not be eliminated completely. This will be further discussed below.

\subsection{Asymptotic properties}

To get some initial insight into the asymptotic properties of the algorithm the load and measurement disturbances are initially neglected. Provided that the iterative procedure converges the asymptotic error signal, using equation (8), becomes

$$
E_{\infty}\left(e^{i \omega T}\right)=\frac{1-Q\left(e^{i \omega T}\right)}{1-Q\left(e^{i \omega T}\right)\left(1-L\left(e^{i \omega T}\right) T_{u}\left(e^{i \omega T}\right)\right)} \tilde{E}\left(e^{i \omega T}\right)
$$

where $E_{\infty}$ and $\tilde{E}$ denote the Fourier transforms of the asymptotic and final error respectively. It is clearly seen that by using $Q \neq 1$ it is impossible to eliminate the error completely, but as will be seen later there are other advantages with this choice. Typically $Q$ is chosen as a low pass filter with unit gain for low frequencies which implies that the low frequency part of $\tilde{e}$ can be reduced substantially. 


\section{Load Disturbances}

A number of observations concerning the handling of load disturbances can be made using equation (8). Consider first the case $Q \equiv 1$ and $n_{k} \equiv 0$, which means that there are no measurement disturbances. This implies the update equation

$$
e_{k+1}=\left(1-L T_{u}\right) e_{k}+T_{d}\left(d_{k}-d_{k+1}\right)
$$

The load disturbances contribute to the error by their differences between the iterations. If a disturbance is of repetitive nature in the sense that the disturbance signals $d_{k}(t)=d_{k+1}(t)$ for all $k$, the contribution to the error difference equation is zero. This assumption is likely for the load disturbance where for example load disturbances due to gravitational forces can be expected to be rather similar during different iterations.

Consider also the situation when $Q \neq 1$, there are no measurement disturbances and $d_{k}(t)=d(t) \forall k$. This corresponds to the error difference equation

$$
e_{k+1}=Q\left(1-L T_{u}\right) e_{k}+(1-Q) \tilde{e}+T_{d}(Q-1) d
$$

The load disturbance will act as a driving term similar to the initial error $\tilde{e}$. In a typical case $T_{d}$, and the filter $Q$ are both of low pass type, which means that $1-Q$ is of high pass type. Multiplying these two will give a band pass filter with a pass band around the bandwidth of the closed loop system. The possibilities to reduce the effects of the load disturbance hence depends on the relationship between the frequency content of the load disturbance and the cut-off frequency of the filter $Q$.

Using equation (16) the asymptotic error, similar to (14), becomes

$$
E_{\infty}\left(e^{i \omega T}\right)=\frac{1-Q\left(e^{i \omega T}\right)}{1-Q\left(e^{i \omega T}\right)\left(1-L\left(e^{i \omega T}\right) T_{u}\left(e^{i \omega T}\right)\right)}\left(\tilde{E}\left(e^{i \omega T}\right)-T_{d}\left(e^{i \omega T}\right) D\left(e^{i \omega T}\right)\right)
$$

where $D$ denotes the Fourier transform of the load disturbance. This expression clearly illustrates the observation above that the frequency content of $d$, the cut-off frequencies of $T_{d}$ and $1-Q$ respectively will determine how well the ILC algorithm handles load disturbances of repetitive character.

\section{Measurement Disturbances}

Measurement disturbances are more likely to be of random character which means that $n_{k+1}(t) \neq n_{k}(t)$ in general. There will hence always be driving 
terms on the right hand side of equation (8) that prevent $e_{k}$ from tending to zero. In order to concentrate on the influence of the measurement disturbance it is here assumed that $r \equiv 0$ and $d \equiv 0$. This implies that $\tilde{e} \equiv 0$, and using equation (1) this gives that

$$
e_{k}=-T_{u} u_{k}-T_{n} n_{k}
$$

The second term on the right hand side is caused by the conventional feedback, and since this term is always present the interest from an ILC viewpoint is on the first term. Equations (5) and (1) together with Figure 1 give

$$
u_{k+1}=Q\left(1-L T_{u}\right) u_{k}-Q L S n_{k}
$$

where $S$ is the transfer function

$$
S=\frac{1}{1+F G}
$$

Assume then that $n_{k}$ is a stationary stochastic process with spectral density $\Phi_{n}(\omega)$, and let, in stationarity, $\Phi_{u}(\omega)$ denote the spectral density of $u_{k}$. Equation (19) then gives

$$
\Phi_{u}\left(e^{i \omega T}\right)=\frac{|Q L S|^{2}}{1-\left|Q\left(1-L T_{u}\right)\right|^{2}} \Phi_{n}\left(e^{i \omega T}\right)
$$

Hence the spectral density of error in equation (18) becomes

$$
\Phi_{e}\left(e^{i \omega T}\right)=\frac{\left|T_{u} Q L S\right|^{2}}{1-\left|Q\left(1-L T_{u}\right)\right|^{2}} \Phi_{n}\left(e^{i \omega T}\right)+\left|T_{n}\right|^{2} \Phi_{n}\left(e^{i \omega T}\right)
$$

It is clear from equation (22) that the magnitude of the error spectral density will be very large for frequencies where $\left|Q\left(1-L T_{u}\right)\right|$ is close to one. As an illustration let $L=\hat{T}_{u}^{-1}\left(1-H_{B}\right)$ where $H_{B}$ is a high-pass filter determining the desired convergence properties of the ILC algorithm and $\hat{T}_{u}$ is a model of the transfer function $T_{u}$. The left plot in Figure 2 shows the principal behavior of $\left|Q\left(1-L T_{u}\right)\right|$ when $Q=1$ while the right plot shows the same quantity when $Q$ is a low pass filter. It is clear that when the $Q$ filter is introduced the high frequency disturbance impact can be reduced.

It is seen that by introducing $Q$ it is possible to reduce the measurement disturbance impact on the ILC algorithm, but with the price that the error in the trajectory following will not tend to zero. The demand of trajectory following and disturbance reduction will in this sense be contradictory. 

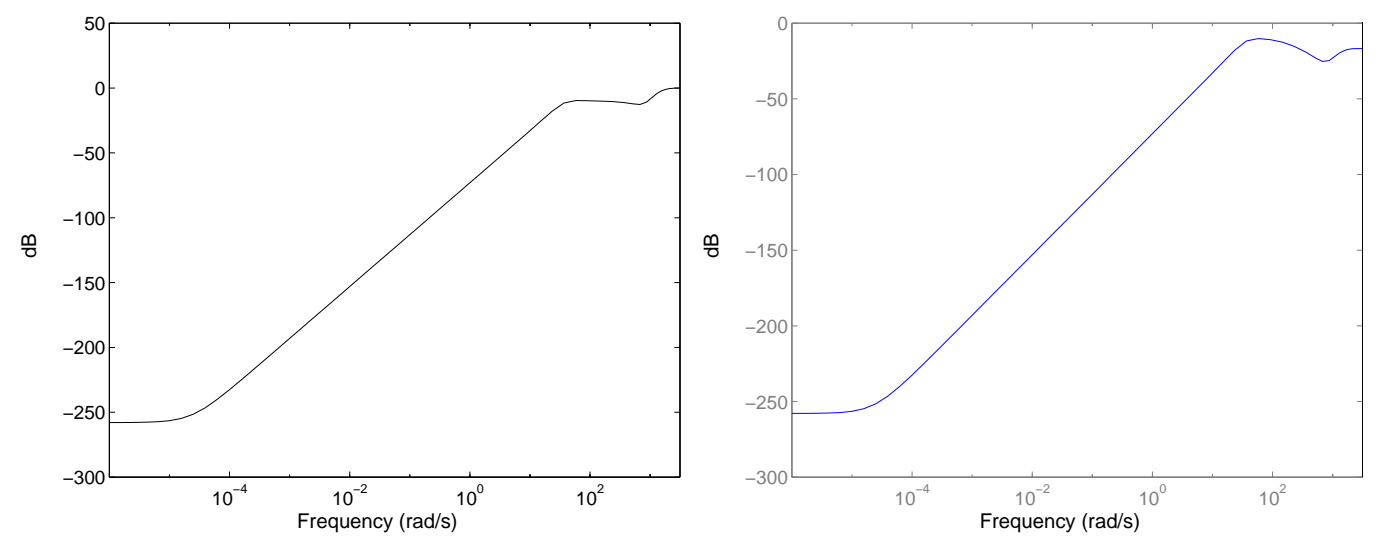

Fig. 2. $\left|Q\left(e^{i \omega T}\right)\left(1-L\left(e^{i \omega T}\right) T_{u}\left(e^{i \omega T}\right)\right)\right|$. Left: $Q\left(e^{i \omega}\right) \equiv 1$. Right: $Q\left(e^{i \omega T}\right)$ low-pass filter.

\section{Simulations}

To illustrate the disturbance properties a simulation example will be studied. The example is a simplified description of a single robot joint modeled as a double integrator. The discrete time representation is given by

$$
G(z)=\frac{T^{2}(z+1)}{2 J(z-1)^{2}}
$$

where $J=0.0094$ is the moment of inertia. The system is controlled by the discrete time PD-regulator

$$
F(z)=K_{P}+\frac{K_{D}}{T} \frac{(z-1)}{z}
$$

where $K_{P}=12.7$ and $K_{D}=0.4$. The feed-forward filter is a double differentiation represented by

$$
F_{f}(z)=\frac{J^{*}(z-1)^{2}}{T^{2} z^{2}}
$$

where $J^{*}$ is the assumed moment of inertia. The model is simulated using 1 $\mathrm{kHz}$ sampling frequency. The applied reference trajectory shown in Figure 3.

The filter $L$ is chosen in a heuristic, but model based, way by choosing

$$
L=\hat{T}_{u}^{-1}\left(1-H_{B}\right)
$$

where $\hat{T}_{u}$ denotes a nominal closed loop transfer function obtained using the model of the open loop system. It is assumed that there is a $30 \%$ error in $J^{*}$, i.e. the moment of inertia in the model of the system. The filter $H_{B}$ is a Butterworth high pass filter (here of second order) for which the gain tends to one for high frequencies. Choosing $L$ according to this design rule, with cut-off 

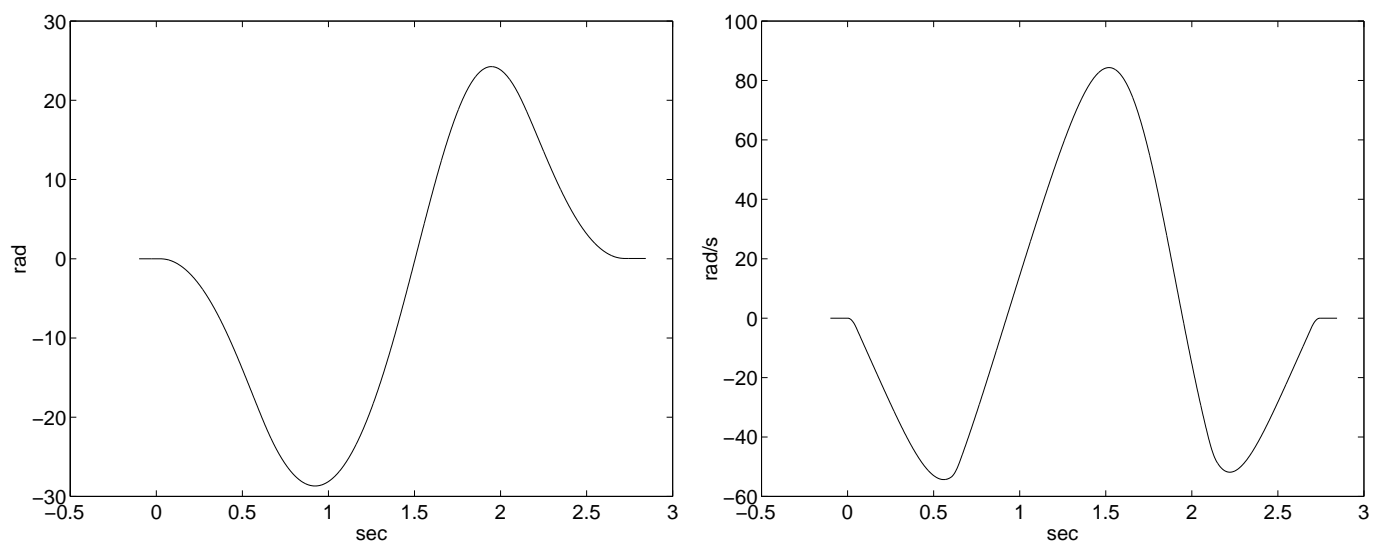

Fig. 3. Left: Reference position $r$. Right: Reference velocity, $\dot{r}$.

frequency of the high pass filter equal to 0.4 times the Nyquist frequency, gives the Nyquist curve depicted in Figure 4, which also shows $T_{u}$ for comparison. Figure 4 also shows the right hand side of the convergence criterion (13) for $Q=1$ and $Q$ chosen as a low pass filter respectively.

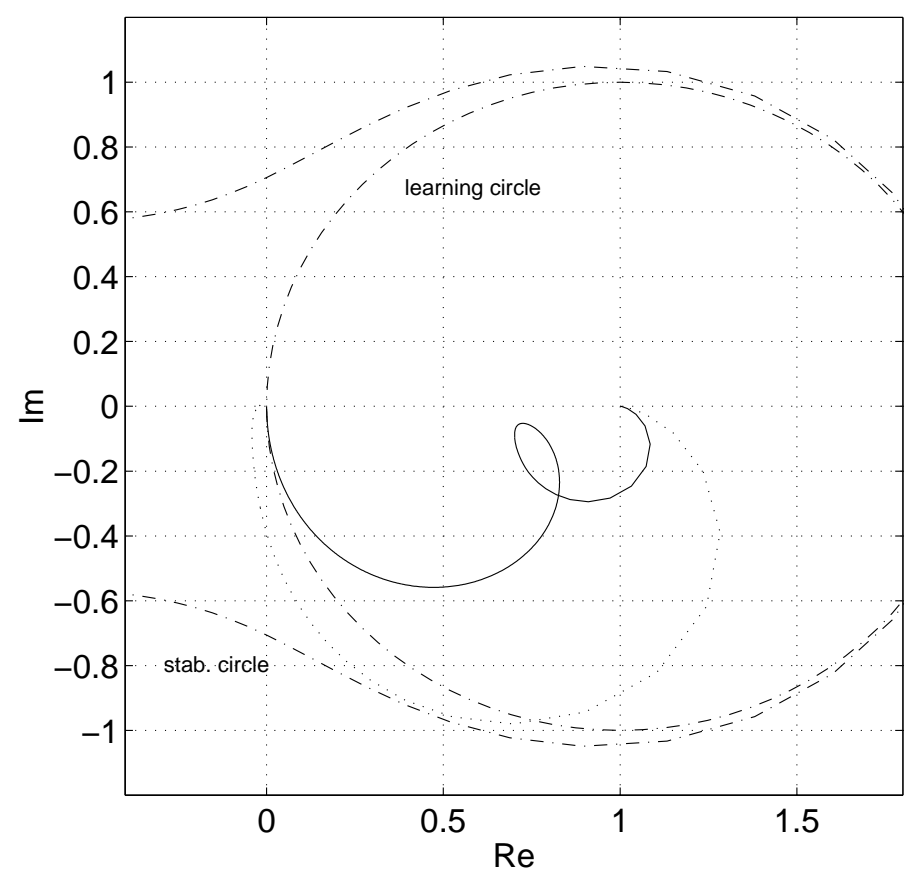

Fig. 4. Dotted: $T_{u}$. Solid: $T_{u} L$ for $L$ designed using a nominal model. Dash-dotted: $1 /\left|Q\left(e^{i \omega T}\right)\right|$ for $Q=1$ and $Q$ chosen as a low pass filter respectively.

The first goal is to investigate how the load disturbance influences the ILC algorithm. The left part of Figure 5 shows the applied load disturbance as a function of time. This disturbance signal is applied at the same time every cycle as a repetitive disturbance. The right part of Figure 5 shows the maximum error for each iteration with and without the $Q$-filter.

Figure 6 shows the spectrum of $E_{k}$ as a function of iteration. Clearly the 

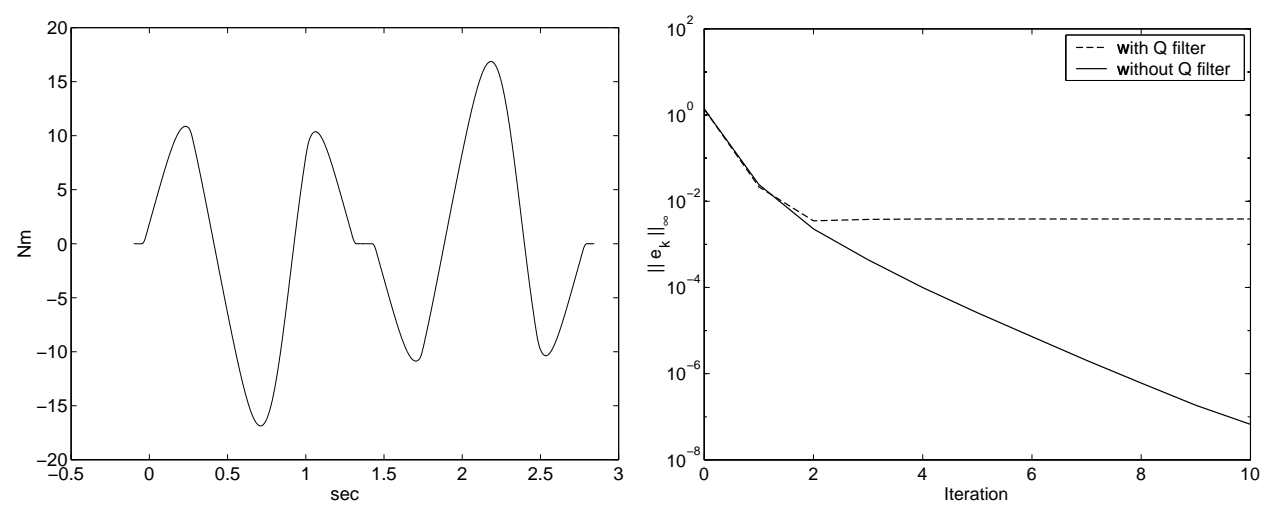

Fig. 5. Left: Load disturbance. Right: Max error vs. iteration.

introduction of a filter $Q$ reduces the convergence speed of the ILC algorithm and there will also not be convergence to zero of the error. Already after one iteration when the filter $Q$ is used the energy in the error has almost reached its final value and also in the spectrum it is possible to see that there is not much change after the first iteration.

The next step is to introduce a measurement disturbance, $n_{k}$, and this is chosen as discrete time white noise process with standard deviation $3 \cdot 2 \cdot 10^{-4}$. The time domain behavior is shown in Figure 7 while the frequency domain properties are shown in Figure 8. Figure 7 shows the error signal energy and the maximum error as function of iteration. The plot of the energy clearly illustrates that the use of $Q$ reduces the error energy caused by the measurement disturbance. The fact that the maximum error behaves in a similar fashion in the two cases is explained by that the maximum error is of low frequency character and hence not affected by the choice of $Q$. In Figure 8 the spectrum of the position error signal $r-z_{k}$ is shown with and without $\mathrm{Q}$ filter. The reduction of the high frequency part of the error spectrum is clearly shown.

\section{Experiments}

The results presented in the previous sections will now be illustrated using an ABB IRB1400 industrial robot. For a more thorough description of the technical part of the experimental setup see (Norrlöf 1998).

In this example ILC is applied to three joints. The robot has a total of 6DOF but for the three wrist joints ILC is not applied. Each of the joints are modeled as a transfer function description from the ILC control input to the measured motor position on the robot. The feedback controller, implemented by $\mathrm{ABB}$, is working in parallel with the ILC and since the controller is working well, the closed loop from reference angular position to measured angular can be described using a low order linear discrete time model. The models are 

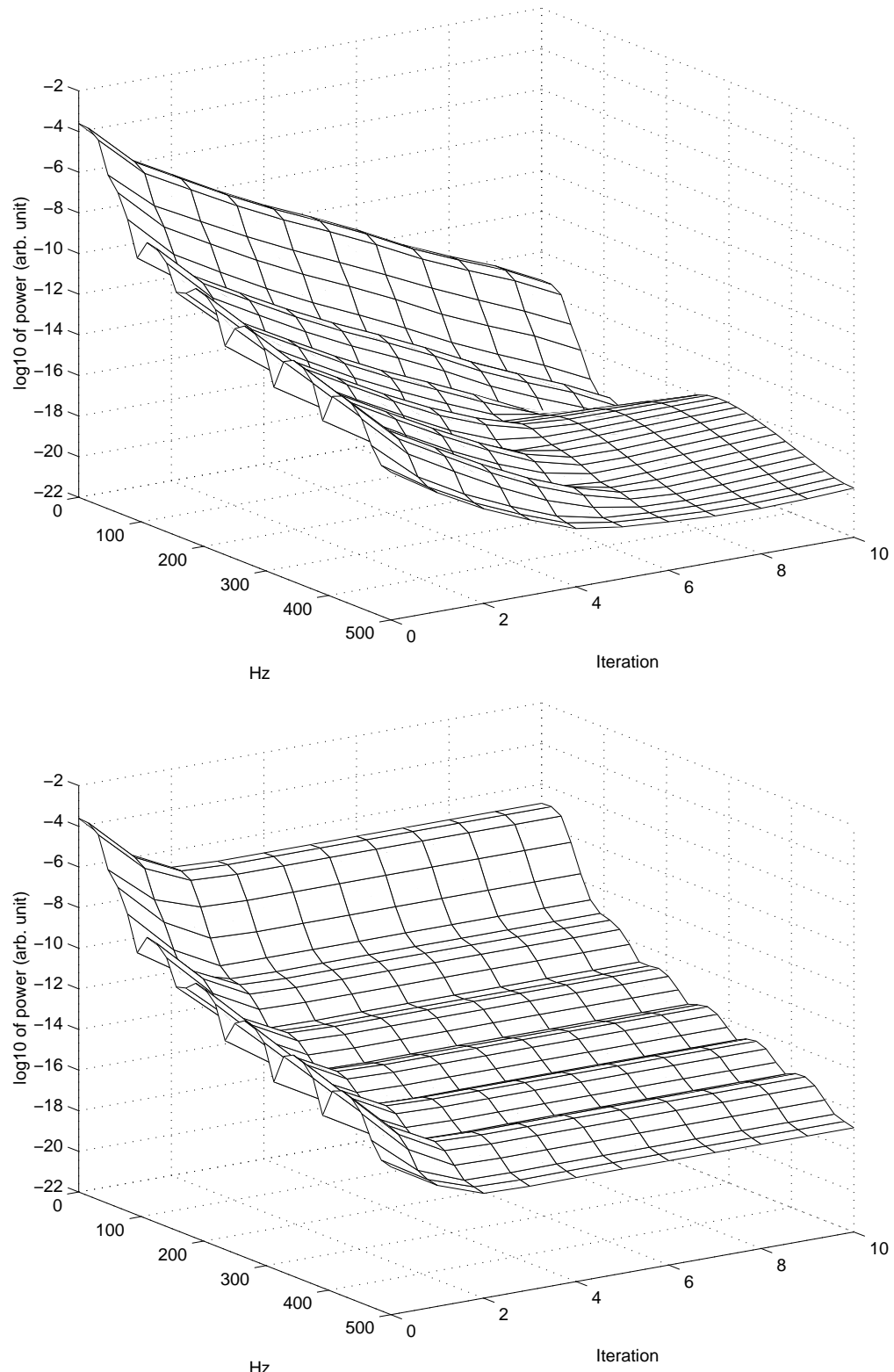

Fig. 6. Upper: Error signal spectrum without Q filter. Lower: Error signal spectrum with Q filter.

obtained by applying system identification to the closed loop system.

In Figure 9 the program used in the experiment is shown together with the resulting trajectory on the arm-side of the robot. Note that the program in Figure 9 is not syntactically exactly the same as the true program. Semantically, however, the programs are the same. The instruction moveLp2, v100, z1 refers to an instruction that produces a straight line on the arm-side of the robot. The line starts from the current position, not explicitly stated, and ends in p2. The speed along the path is in this case programmed to be $100 \mathrm{~mm} / \mathrm{s}$. The last parameter $\mathrm{z} 1$ indicates that the point $\mathrm{p} 2$ is a zone point. this means 

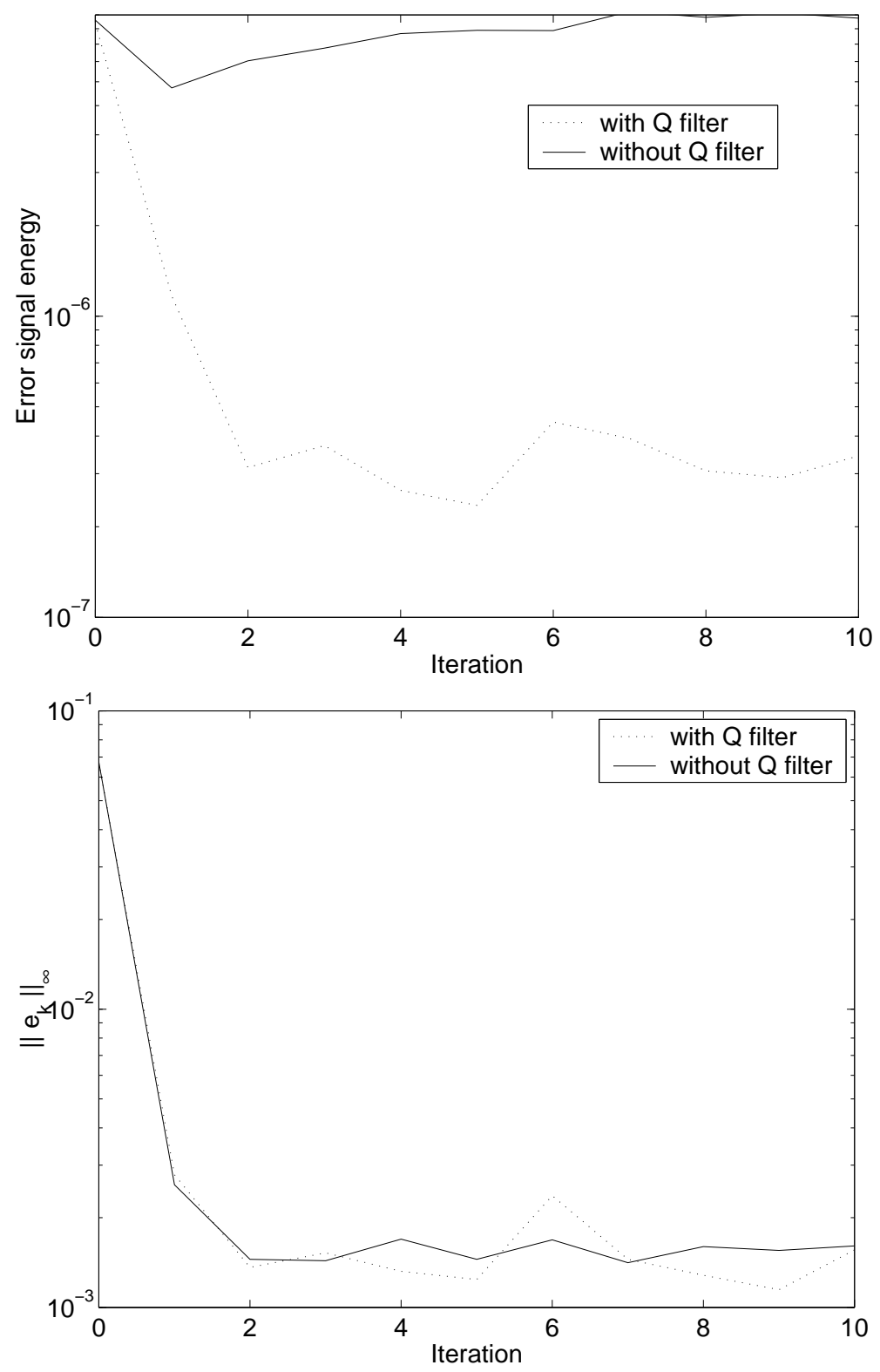

Fig. 7. Upper: Error signal energy. Lower: Maximum absolute position error.

that the robot will pass in a neighborhood of the point with a distance not more than $1 \mathrm{~mm}$. This can also be seen in Figure 9. The moveL instruction is a simplified version of the corresponding instruction MoveL in the RAPID programming language The actual position of $\mathrm{p} 1$ in the base coordinate system is $\mathrm{x}=1300 \mathrm{~mm}, \mathrm{y}=100 \mathrm{~mm}$, and $\mathrm{z}=660 \mathrm{~mm}$.

The aim of the experiment is to illustrate the importance of the $Q$-filter with respect to measurement disturbances. Since experiments on real systems always will contain measurement disturbances it will not be possible to compare the performance with a situation without measurement disturbances. The aim will therefore be to illustrate the positive influence of the introduction of the 

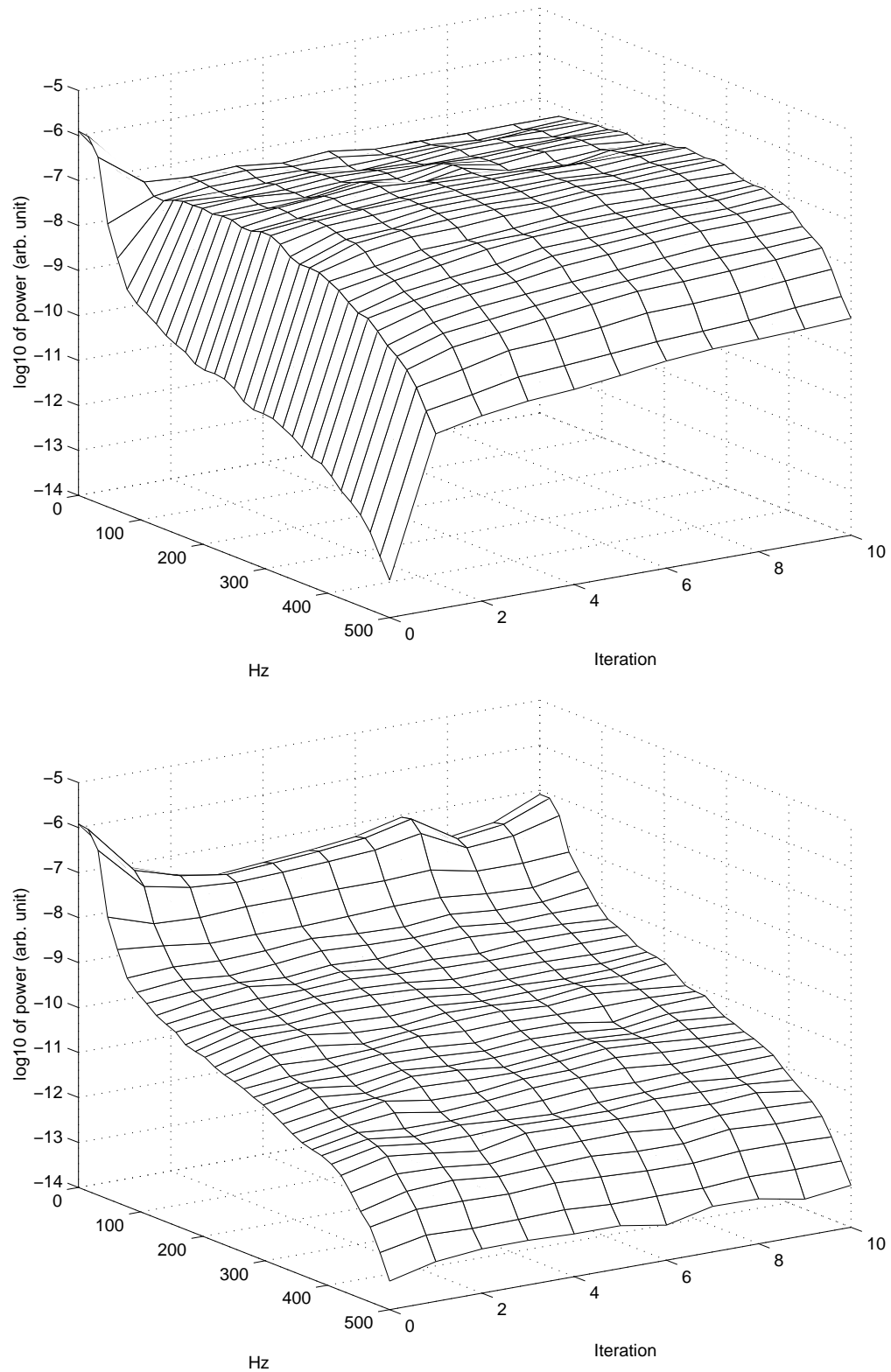

Fig. 8. Upper: Error signal spectrum, without Q filter. Lower: Error signal spectrum with Q filter.

filter $Q$. In the experiments the filter $L$ is chosen as $L(z)=0.9 z^{4}$ i.e. a scaling times a time shift four samples forward. This hence corresponds to a noncausal filtering. This choice gives moderate performance requirements. The ILC algorithm is run during ten iterations with $Q=1$ and $Q$ chosen as a low pass filter respectively. Figure 6 illustrates the performance for the three motors involved. Choosing $Q$ as a low pass filter the error settles at a steady level while the choice $Q=1$ gives large fluctuations in the error. It should be noted that the conditions in the experiments differs from the simulation since the undisturbed position error $z_{k}$ is not available. Instead the measured position error $r-y_{k}$ has to be used for evaluation. 
$\%$ starting at $\mathrm{p} 1$ moveL p2,v100,z1;

moveL p3,v100,z1;

moveL p4,v100,z1;

moveL p5,v100,z1;

moveL p6,v100,z1;

movel p1,v100,fine;

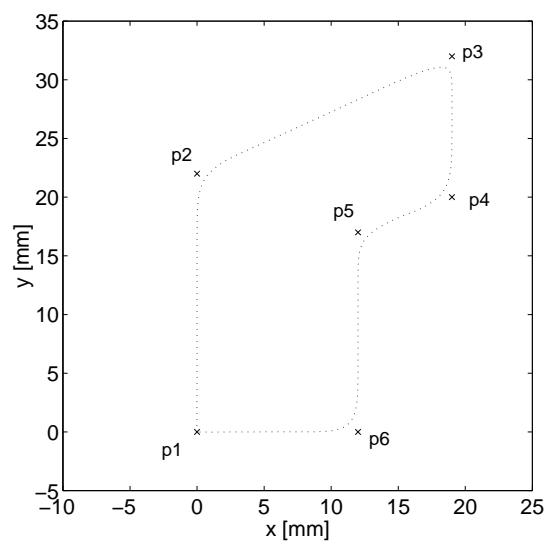

Fig. 9. The program used to produce the trajectory used in the example (left) and the resulting trajectory on the arm-side translated such that $\mathrm{p} 1$ is in the origin (right).
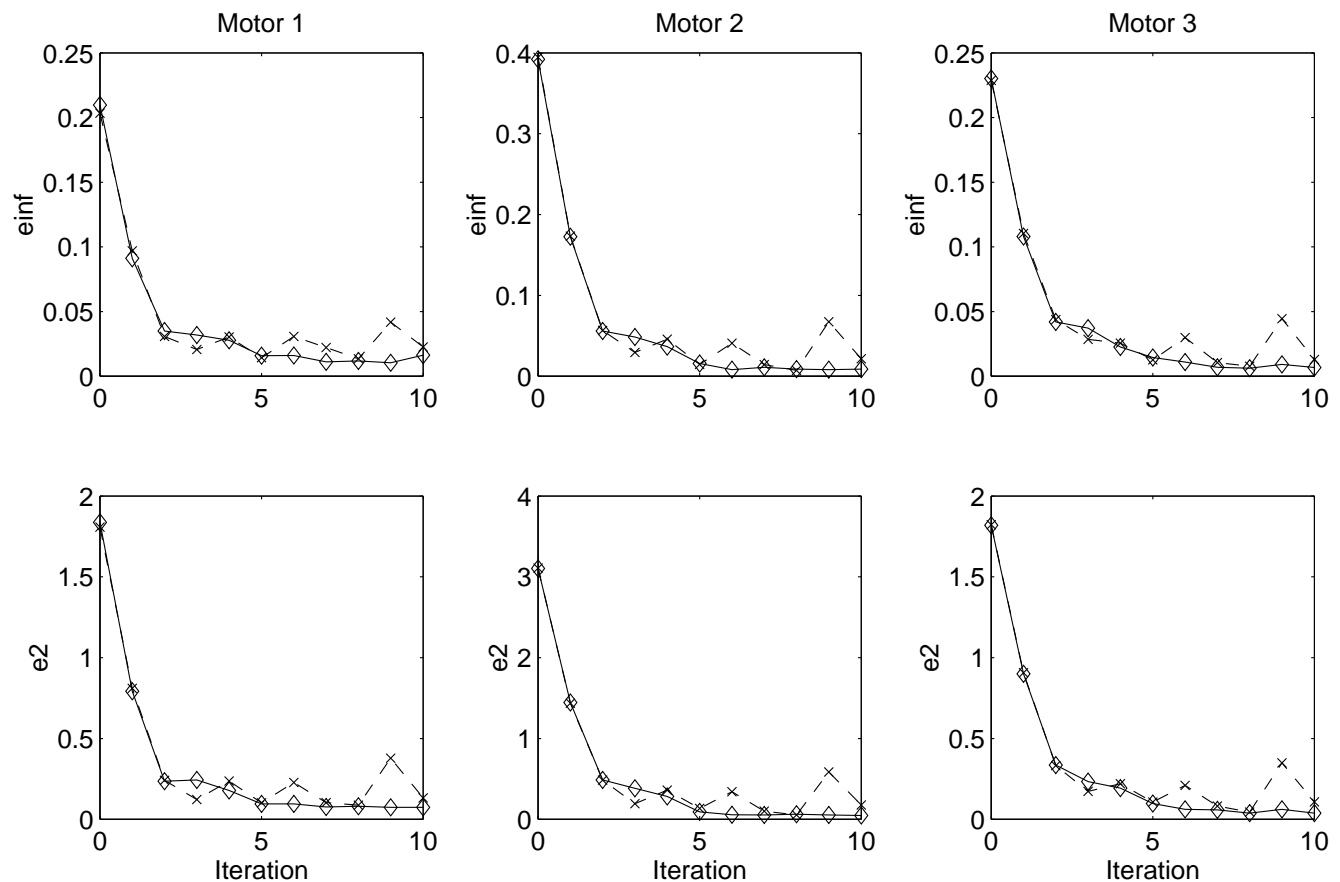

Fig. 10. Upper: Maximum error for each motor. Lower: Error energy.

\section{Conclusions}

Disturbance aspects of Iterative Learning Control algorithm has been studied using frequency domain methods. It has been how the choice of design filters, in particular the filter $Q$, affects the way load and measurement disturbances affect the performance of ILC algorithms. The results have been supported by 
simulations and experiments on a real robot.

\section{References}

Arimoto, S., S. Kawamura and F. Miyazaki (1984). "Bettering Operation of Robots by Learning". Journal of Robotic Systems pp. 123-140.

Bien, Z. and J.X. Xu (1998). Iterative Learning Control. Analysis, Design, Integration and Applications. Kluwer Academic Publishers. Dordrecht, The Netherlands.

Craig, J. (1988). Adaptive Control of Mechanical Manipulators. Addison-Wesley Publishing Company.

de Roover, D. (1996). "Synthesis of a Robust Iterative Learning Controller Using an $H_{\infty}$ approach". In: Proc. of the 35th IEEE Conference on Decision and Control. Kobe, Japan. pp. 3044-3049.

Gorinevsky, D.M., D. Torfs and A.A. Goldenberg (1995). "Learning approximation of feedforward dependence on the task parameters: Experiments in direct-drive manipulator tracking”. In: Proc. ACC 1995. Seattle, Washington. pp. 883-887.

Gunnarsson, S. and M. Norrlöf (1997). "Some Experiences of the Use of Iterative Learning Control for Performance Improvement in Robot Control Systems". In: IFAC Symposium in Robot Control 1997. Nantes, France. pp. 379-383.

Hideg, L. (1992). Stability of Learning Control Systems. PhD thesis. Oakland University. Rochester, Michigan.

Horowitz, R. (1993). "Learning Control of Robot Manipulators". ASME Journal of Dynamic Systems, Measurement, and Control 115, 403-411.

Moore, K.L. (1993). Iterative Learning Control for Deterministic Systems. Springer Verlag.

Moore, K.L. (1998). "Iterative Learning Control: An Expository Overview". Applied and Computational Controls, Signal Processing and Circuits.

Norrlöf, M. (1998). On analysis and implementation of iterative learning control. Licentiate thesis LIU-TEK-LIC-1998:62 Linköping Studies in Science and Technology. Thesis No 727. Department of Electrical Engineering, Linköpings universitet.

Panzieri, S. and G. Ulivi (1995). "Disturbance rejection of Iterative Learning Control Applied to Trajectory for a Flexible Manipulator". In: Proc. ECC 1995. Rome, Italy. pp. $2374-2379$. 